\title{
Afghanistan als Ende des liberalen Moments?
}

\author{
Tobias Bunde, Timo Noetzel und Adrian Oroz*
}

\begin{abstract}
Debates concerning the Afghanistan intervention rarely address the rather abstract yet essential question of what impact a potential 'defeat' in Afghanistan might have on the 'liberal moment' in world politics. The past two decades have been dominated by liberal values, which are based on the rights of the individual. If the 'international community' withdraws from Afghanistan without having established a political order that fulfills fundamental liberal requirements, the long-term consequences for the idea of an 'international community' and the universality of liberal values would be severe, especially in cases where, unlike in Afghanistan, vital security interests are not considered to be at stake.
\end{abstract}

Keywords: Afghanistan, world order, liberal values, international community

Afghanistan, Weltordnung, liberale Werte, internationale Gemeinschaft

\section{Einleitung}

$\mathrm{I}$ $\mathrm{n}$ den vergangenen Monaten haben vor allem Debatten um die Möglichkeit eines „Scheiterns“ und um einen Rückzug der internationalen Gemeinschaft aus Afghanistan den Afghanistan-Diskurs in den westlichen Öffentlichkeiten geprägt. Im Gegensatz zu einigen anderen Ländern spielen in Deutschland zwei zentrale Aspekte nur eine untergeordnete Rolle: zum einen die möglichen Konsequenzen eines Rückzugs internationaler Akteure für die afghanische Bevölkerung, ${ }^{1}$ zum anderen die Bedeutung eines Scheiterns für die Wertgrundlage der „internationalen Gemeinschaft", letztlich somit die Frage nach der Zukunft internationaler Ordnungspolitik. ${ }^{2}$ Betrachtet man den Afghanistaneinsatz unter dem Blickwinkel der normativen Entwicklungen der letzten Jahrzehnte, so haben aber genau diese beiden Aspekte eine besondere Relevanz. Schließlich ergeben sich einige grundlegende Fragen: Würden mit einem möglichen Scheitern in Afghanistan auch die liberalen Ordnungsvorstellungen einen Rückschlag erleiden, die in den letzten zwei Jahrzehnten das Handeln der internationalen Gemeinschaft geprägt haben? Welche Bedeutung hätte ein Scheitern in Afghanistan für die weitere Entwicklung der internationalen Gemeinschaft? Und welche Rolle werden Menschenrechte für die westliche Außenpolitik in Zukunft spielen?

* Tobias Bunde, M.A., ist Doktorand und assoziiertes Mitglied der Nachwuchsgruppe „Konfliktgeneratoren“ im Exzellenzcluster „Kulturelle Grundlagen von Integration“ an der Universität Konstanz. Dr. Timo Noetzel ist Leiter der Nachwuchsgruppe „Konfliktgeneratoren“ und Fellow der Stiftung Neue Verantwortung in Berlin. Adrian Oroz, M.A., ist freiberuflicher Politikwissenschaftler. Die Autoren danken zwei anonymen Gutachterinnen bzw. Gutachtern für wertvolle Anregungen.

1 So betont etwa Thomas Ruttig, einer der profiliertesten deutschen Afghanistankenner, dass Medien in den englischsprachigen Ländern sich auch bemühen, , darüber zu berichten, wie es der afghanischen Bevölkerung bei all dem geht. In Deutschland konzentriert sich viel zu viel auf die Bundeswehr." Eher Rückschritte als Fortschritte, Interview mit Thomas Ruttig, in: Islamische Zeitung, 26.8.2010, http://www.islamische-zeitung.de/?id=13661 (1.9.2010). Auch bei der Strategiediskussion liegt der Fokus der Debatte meist allein auf der Bundeswehr, während die zivilen Instrumente trotz des grundsätzlichen Konsenses, dass der Konflikt nicht allein auf militärischem Wege beendet werden kann, kaum Berücksichtigung finden. Vgl. z.B. Timo Noetzel und Thomas Rid, Mehr Köche als Diplomaten, in: Financial Times Deutschland, 5.2.2010, S. 26

2 Als Ausnahme vgl. aber z.B. Thomas Risse, Was in Afghanistan auf dem Spiel steht, in: Internationale Politik, Vol. 62, No. 4, 2007, S. 106-108; Thomas Assheuer, Am Ende. Der Westen scheitert in Afghanistan. Ist damit die Allgemeingültigkeit der Menschenrechte widerlegt?, in: Die Zeit, 15.4.2010, S. 45, für den ein Scheitern der ISAF auch „eine Niederlage auf dem Kampfplatz der Ideen" wäre.

\section{Der "liberale Moment" und die Ordnungspoli- tik der „internationalen Gemeinschaft“}

Nachdem der konservative Kolumnist Charles Krauthammer schon 1991 den Begriff des ,unipolaren Moments“3 geprägt hatte, um die Einzigartigkeit der US-amerikanischen Übermacht zu beschreiben, ist die Zeit seit dem Ende des Ost-West-Konflikts häufig als die einer unipolaren Weltordnung beschrieben worden. Eine solche verengte Fokussierung auf die wirtschaftliche und vor allem militärische Vormachtstellung der USA verkennt jedoch viel weiter reichende Entwicklungen, die die internationale Ordnungspolitik vor allem seit den 1990er Jahren geprägt haben. ${ }^{4}$ Man muss Francis Fukuyamas oft missverstandene These vom „Ende der Geschichte“ ${ }^{\text {5 }}$ nicht uneingeschränkt teilen, um zumindest zu akzeptieren, dass liberale Ordnungsvorstellungen - mit der Freiheit und Selbstbestimmung des Individuums als normativem Ausgangspunk $\mathrm{t}^{6}$ - nach dem Ende des OstWest-Konflikts eine bis dahin ungekannte Dominanz erlangten und die Vorstellung einer „internationalen Gemeinschaft“, die mehr sein sollte als eine lose Gemeinschaft souveräner Staaten, maßgeblich prägten. Wenngleich der Optimismus der Anfangsjahre - die Hoffnung auf eine „neue Weltordnung“ und die Zwangsläufigkeit fortschreitender Demokratisierung - mittlerweile verblaßt ist, haben liberale Werte Weltordnungspolitik in einem solchen Maße geprägt, dass es in der Tat angemessen

3 Charles Krauthammer, The Unipolar Moment, in: Foreign Affairs, Vol. 70, No. 1, 1991, S. 23-33.

4 Siehe dazu auch Ted Hopf, Globalizing the Authoritative Alliance, in: Mathias Jopp und Hanna Ojanen (Hg.), European Security Integration. Implications for Non-alignment and Alliances, Helsinki 1999, S. 191-213.

5 Francis Fukuyama, Das Ende der Geschichte. Wo stehen wir?, München 1992.

6 Vgl. die Definition bei Michael W. Doyle, Kant, Liberal Legacies, and Foreign Affairs, in: Philosophy and Public Affairs, Vol. 12, No. 3, 1983, S. 205-235, S. 206f., der hier gefolgt werden soll: „A commitment to a threefold set of rights forms the foundation of liberalism. Liberalism calls for freedom from arbitrary authority, often called ,negative freedom, ' which includes freedom of conscience, a free press and free speech, equality under the law, and the right to hold, and therefore to exchange, property without fear of arbitrary seizure. Liberalism also calls for those rights necessary to protect and promote the capacity and opportunity for freedom, the,positive freedoms'. Such social and economic rights as equality of opportunity in education and rights to health care and employment, necessary for effective self-expression and participation, are thus among liberal rights. A third liberal right, democratic participation or representation, is necessary to guarantee the other two. To ensure that morally autonomous individuals remain free in those areas of social action where public authority is needed, public legislation has to express the will of the citizens making laws for their own community." 
ist, nicht nur von einem unipolaren, sondern auch von einem liberalen Moment ${ }^{7} \mathrm{zu}$ sprechen.

Die Entwicklung der Idee der „internationalen Gemeinschaft“ ist insbesondere in den Schriften der sogenannten English School in den Internationalen Beziehungen aufgegriffen worden. ${ }^{8}$ Der pluralistische Strang der English School geht von einer Staatengemeinschaft aus, die sich auf grundlegende Regeln internationaler Ordnung verständigt, sich aber der Einmischung in die inneren Angelegenheiten ihrer Mitglieder im Allgemeinen enthält, um den zwischenstaatlichen Frieden einer durch kulturelle Unterschiede geprägten Welt nicht zu gefährden. Dagegen betont die solidaristische Ausformung dieser Denktradition gerade die Herausbildung gemeinsamer Werte über Staatsgrenzen hinweg, die den einzelnen Mitgliedern der Gemeinschaft im Sinne eines good international citizenship gewisse Rechte und Pflichten auferlegen. Andrew Hurrell folgend kann die Kernidee des solidarism in den regelmäßig erfolgenden Anrufen der „internationalen Gemeinschaft“ gesehen werden, die als Sinnbild für eine Weltordnung gelten kann, in der die Kluft zwischen Recht und Macht einerseits sowie Recht und Moral andererseits zumindest verringert, wenn auch nie vollständig geschlossen werden kann. ${ }^{9}$

Seit dem Zerfall der bipolaren Ordnung hat sich die Weltordnung zunehmend in Richtung eines liberal solidarism entwickelt. Dahinter steht zunächst der Aufstieg der liberalen Demokratie zum weitgehend unumstrittenen normativen Referenzpunkt politischer Ordnung. Die Ergebnisse der World Values Surveys zeigen, dass ein demokratisches Herrschaftssystem von Menschen in allen Teilen der Welt als bevorzugte Regierungsform angesehen wird. ${ }^{10}$ Besonders nachdem die liberale Demokratie mit dem Zerfall der Sowjetunion ihren ideologischen Gegenspieler verloren hatte, fanden liberale Ordnungsvorstellungen in zunehmendem Maße Eingang in die Völkerrechtsordnung als rechtlicher Grundlage der „internationalen Gemeinschaft".${ }^{11}$ Nicht nur in Europa und Ameri$\mathrm{ka}$, sondern auch in anderen Weltregionen entstanden nach

7 Vgl. z.B. Daniel Green, The Lingering Liberal Moment: An Historical Perspective on the Global Durability of Democracy After 1989, in: Democratization, Vol. 6 , No. 2, 1999, S. 1-41, v.a. S. 20.

8 Allerdings gilt es zu beachten, dass die Vertreter der English School von der international society sprechen, nicht von der international community. Zumindest die Anhänger einer solidaristischen international society sind jedoch dem Verständnis des Begriffs der internationalen Gemeinschaft im Deutschen sehr nahe. Im Folgenden wird der Begriff der internationalen Gemeinschaft benutzt, ohne damit eine Präferenz ausdrücken zu wollen. Weiterführend zum Begriff vgl. Andreas Paulus, Die internationale Gemeinschaft im Völkerrecht. Eine Untersuchung zur Entwicklung des Völkerrechts im Zeitalter der Globalisierung, München 2001; Berit Bliesemann de Guevara und Florian P. Kühn, The „International Community“ - Rhetoric or Reality? Tracing a Seemingly Well-known Apparition, in: Sicherheit + Frieden, Vol. 27, No. 2, 2009, S. 73-79; Fabian Schellhaas, Die „internationale Gemeinschaft “ im 21. Jahrhundert - Ein Gespenst geht um..., in: Christian Tomuschat (Hg.), Weltordnungsmodelle für das 21. Jahrhundert. Völkerrechtliche Perspektiven, Baden-Baden 2009, S. 25-64.

9 Andrew Hurrell, On Global Order. Power, Values, and the Constitution of International Society, Oxford 2007, S. 5.

10 Russell J. Dalton, Doh C. Shin und Willy Jou, Understanding Democracy: Data From Unlikely Places, in: Journal of Democracy, Vol. 18, No. 4, 2007, S. 142-156. Natürlich ließe sich hier einwenden, dass die Zustimmung v.a. von der speziellen Ausprägung der „Demokratie“ abhängt. Interessanterweise liegen die Demokratiekonzeptionen aber sehr eng beieinander. Vgl. dazu Christian Welzel und Roland Inglehart, The Role of Ordinary Citizens in Democratization, in: Journal of Democracy, Vol. 19, No. 1, 2008, S. 126-140.

11 Vgl, ausführlich Tobias Bunde, Das Völkerrecht der Demokratien. Ambivalenzen einer liberalen Weltordnung, in: Christian Tomuschat (Hg.), Weltordnungsmodelle für das 21. Jahrhundert. Völkerrechtliche Perspektiven, Baden-Baden 2009, S. 99-162. und nach regionale Vertragswerke, die sich der Förderung und Bewahrung demokratischer Herrschaft verschrieben haben und in ihrer Summe einen „demokratischen Flickenteppich“ darstellen, der bereits weite Teile der Welt bedeckt. ${ }^{12}$ Demokratieförderung und Wahlbeobachtungsmissionen konnten so zu „normalen“ Tätigkeitsfeldern der internationalen Gemeinschaft werden. ${ }^{13}$

Auch für die Vereinten Nationen, in deren Charta das Wort „Demokratie“ nicht genannt wird, ist Demokratie mittlerweile ein Leitbegriff. ${ }^{14} 2005$ wurde beispielsweise die Einrichtung eines UN-Demokratiefonds beschlossen, mit dessen Mitteln seit 2006 staatliche und zivilgesellschaftliche Projekte zur Förderung demokratischer Partizipation unterstützt werden. ${ }^{15}$ In Haiti und Sierra Leone autorisierte der Sicherheitsrat sogar Koalitionen, um die Wiedereinsetzung einer demokratisch gewählten Regierung zu ermöglichen. ${ }^{16}$ Nachdem heute somit selbst das politische System der Staaten in immer stärkerem Maße in den Regelungsbereich des regionalen oder universalen Völkerrechts fällt, bleibt vom ursprünglich so weit gefassten domaine réservé staatlicher Souveränität immer weniger bestehen.

Als Zielvorgabe spielt die Demokratie nicht zuletzt bei den vielen Friedensmissionen eine Rolle, die unter Führung oder im Auftrag der Vereinten Nationen unternommen wurden. Diesem Ziel liegt die Überzeugung zu Grunde, dass marktwirtschaftliche Demokratien Frieden und Sicherheit nach innen und außen hervorbringen. ${ }^{17}$ Zwar folgen die unterschiedlichen Friedensmissionen nicht allesamt einer einzigen Blaupause, aber sie sind doch so eindeutig von liberalen Werten geleitet, dass man von „liberalem Peacebuilding“18 sprechen kann: „Since the 1990s, the donor community has concluded that post-conflict societies must undergo a threefold transition: war to peace, command economy to free market enterprise, and authoritarian system to open political order. " 19 Roland Paris bezeichnet diese Missionen gar als „Transmissionsriemen“, mit dem die internationale Gemeinschaft die Verbreitung liberaler Wertvorstellungen als eine moderne Form des standard of civilization übertragen möchte. ${ }^{20}$ Damit zeichnet sich eine weitreichende

12 Dieses Bild stammt von Edward McMahon und Scott Baker, Piecing a Democratic Quilt? Regional Organizations and Universal Norms, Bloomfield 2006. Übersichten über solche Verträge, Resolutionen und Deklarationen finden sich z.B. bei OHCHR, Compilation of Documents or Texts Adopted and Used by Various Intergovernmental, International, Regional and Subregional Organizations Aimed at Promoting and Consolidating Democracy, Genf 2003.

13 Vgl. zum Bedeutungszuwachs von Wahlbeobachtungen v.a. Judith Kelley, Assessing the Complex Evolution of Norms: The Rise of International Election Monitoring, in: International Organization, Vol. 62, No. 2, 2008, S. 221 255.

14 Als jüngstes Beispiel siehe nur die Guidance Note of the Secretary-General on Democracy von 2009, http://www.un.org/democracyfund/Docs/UNSG\%20G uidance\%20Note\%20on\%20Democracy.pdf (25.5.2010).

15 Vgl. http://www.un.org/democracyfund/.

16 Gregory H. Fox, Democratization, in: David M. Malone (Hg.), The UN Security Council. From the Cold War to the 21st Century, Boulder 2004, S. 69-84, S. 69.

17 Vgl. z.B. Roland Paris, At War's End. Building Peace after Civil Conflict, New York 2004, S. 40-42.

18 Vgl. Michael Barnett, Building a Republican Peace. Stabilizing States After War, in: International Security, Vol. 30, No. 4, 2006, S. 87-112, S. 88; zur Argumentation, dass diese Form zunehmend unter Druck gerät, vgl. Roland Paris, Saving Liberal Peacebuilding, in: Review of International Studies, Vol. 36, No. 2, 2010, S. 337-365.

19 Jeroen de Zeeuw und Krishna Kumar, Democracy Assistance to Postconflict Societies, in: dies. (Hg.), Promoting Democracy in Postconflict Societies, Boulder 2006, S. 1-21, S. 4

20 Roland Paris, International Peacebuilding and the „mission civilisatrice“, in: Review of International Studies, Vol. 28, No. 4, 2002, S. 637-656, S. $653 \mathrm{f}$ 
Weiterentwicklung des klassischen Souveränitätsbegriffs ab, dessen Legitimität zunehmend an die Aufrechterhaltung bestimmter Menschenrechtsstandards geknüpft wird und in der weiterhin umstrittenen Norm der Responsibility to Protect (R2P) seinen bislang stärksten Ausdruck gefunden hat. ${ }^{21}$

Freilich tritt eine solche solidaristische Konzeption der „internationalen Gemeinschaft“, die Menschenrechte vor das Prinzip der Nichteinmischung stellt, das aus Sicht der Pluralisten einen Garanten für internationalen Frieden darstellt, aus Sicht der Solidaristen einen nachhaltigen Frieden jedoch verhindert in der Tagespolitik häufig hinter andere Erwägungen zurück. Darfur ist ein gutes Beispiel dafür, welchen Herausforderungen „solidaristische“ liberale Normen wie die R2P gegenüberstehen. ${ }^{22}$ Doch wenn der Fall Darfur als ein harter Testfall für die Bedeutung und Verteidigung liberaler Werte und „internationaler Solidarität" gelten kann, müsste Afghanistan eher ein leichter Fall sein, zum einen deshalb, weil der Einsatz dort stets mit der Verteidigung vitaler Sicherheitsinteressen gerechtfertigt wird, zum anderen aufgrund des betriebenen Aufwands seitens der an ISAF beteiligten Länder. Sollten die in Afghanistan engagierten Akteure tatsächlich beschließen, das Land zu verlassen, ohne sicher zu sein, Fortschritte z. B. im Bereich der Frauenrechte langfristig gesichert zu haben, wäre ein Eingreifen aus primär humanitären Motiven in Ländern von geringerer strategischer Relevanz und eine Verteidigung liberaler Werte dort zukünftig erst recht zunehmend unwahrscheinlich.

\section{Afghanistan und der liberale Moment}

Auf den ersten Blick hat die westliche Intervention in Afghanistan wenig mit dem liberalen Moment und der Verbreitung liberaler Ordnungsvorstellungen zu tun. Ohne die Unterstützung der Taliban für al-Qaida im Vorfeld der Anschläge vom 11. September 2001 hätte es aller Wahrscheinlichkeit nach keine Intervention gegeben. Geradezu selbstverständlich wurde danach jedoch eine Demokratisierung des Landes in Angriff genommen und eine legitime, rechtsstaatliche und demokratische Ordnung als Ziel ausgerufen. Die Frage, ob man in Afghanistan eine Art Alleinherrscher installieren könnte, stellte sich nicht. Man handelte nach der liberalen Überzeugung, dass langfristig nur Demokratien stabil und friedfertig sind, nach innen wie nach außen - diesem Credo entsprechend ist es „nicht illusorisch, Afghanistan demokratisieren zu wollen; vielmehr ist es illusorisch, Afghanistan ohne Demokratisierung befrieden zu wollen." 23

Im Laufe der Jahre sind die externen Akteure in ihren Zielvorgaben zurückhaltender geworden. Die massiven Wahlfälschungen bei den Präsidentschaftswahlen 2009 wurden mit Empörung zur Kenntnis genommen, blieben aber mangels Alternative weitgehend folgenlos. Selbst ursprünglich noch

21 Vgl. als Überblick Andreas von Arnauld, Souveränität und Responsibility to Protect, in: Die Friedens-Warte, Vol. 84, No. 1, 2009, S. 11-52.

22 Siehe zum Fallbeispiel Darfur für die Debatte zwischen Pluralisten und Solidaristen David R. Black und Paul D. Williams, Introduction. International Society and the Crisis in Darfur, in: dies. (Hg.), The International Politics of Mass Atrocities. The Case of Darfur, London 2010, S. 1-23.

23 Patrick Keller, Argumente für Afghanistan: Ein Leitfaden durch die deutsche Debatte, KAS Analysen und Argumente 66, Juni 2009, S. 6. strikt ausgeschlossene Vorgehensweisen wie möglicherweise weitreichende Zugeständnisse an radikale Kräfte werden nun ins Auge gefasst. Offensichtlich ist, dass damit auch eine Neubewertung der Ziele einhergehen müsste. Von einigen wird dies sogar explizit gefordert: „Die Chance, das Land in absehbarer Zeit nicht als Verlierer verlassen zu müssen, besteht allein in der Normabrüstung des Westens. " 24 Auch im Vorwort zum Friedensgutachten 2010 heißt es - mit einer guten Spur Resignation: „Wie es scheint, muss sich der Westen darauf einstellen, in Sachen Demokratie- und Menschenrechtsstandards einen hohen Preis für die Stabilisierung dieses Landes zu zahlen - falls sie überhaupt noch möglich ist. " 25

Diesen Preis wird aber vor allem die afghanische Bevölkerung entrichten müssen, wie der Theologe Gerhard Beestermöller warnt: „Das Schlimmste, was für die Wehrlosen eintreten kann, ist eine (Un-)Ordnung, in der sie gänzlich rechtlos dem Gutdünken der Mächtigen ausgeliefert sind. Daher muss ein Minimum an Rechtszustand erreicht werden, bevor die Truppen Afghanistan verlassen dürfen. " 26 Afghanische Politikerinnen bringen daher ihre Sorge zum Ausdruck, dass vor allem die Rechte der Frauen einer solchen Normabrüstung zum Opfer fallen könnten. „Women's rights must not be the sacrifice by which peace is achieved“, warnt etwa Fawzia Koofi, ehemalige Vizesprecherin des Parlaments. ${ }^{27}$ Auch die Generalsekretärin von Amnesty International Deutschland mahnt, es dürfe keinen „Ausverkauf der Menschenrechte an die Taliban“28 geben.

Zwar sprechen einige Gründe dagegen, Afghanistan zum Testfall für die Zukunft liberaler Ordnungspolitik auszurufen; schließlich kann man die ISAF-Mission als einen besonders komplexen Einzelfall begreifen. ${ }^{29}$ Aber dennoch wirft gerade der Afghanistaneinsatz die Frage auf, in welchem Maße westliche Länder in Zukunft bereit sein werden, bedeutende Ressourcen finanzieller und personeller Art bereitzustellen, wenn sie dies nicht einmal in einem Fall tun, dessen Ausgang von vielen Mitgliedern der internationalen Gemeinschaft als entscheidend für die eigene nationale Sicherheit dargestellt wird. In ihrer Regierungserklärung vom 22. April 2010 fasste Bundeskanzlerin Angela Merkel die Einschätzung der Bundesregierung zusammen: „Wir dürfen niemals vergessen, worum es für uns in Afghanistan geht: [...] Es geht um die Sicherheit Deutschlands, die Sicherheit Europas, die Sicherheit unserer Partner in der Welt, die auch am Hin-

24 Herfried Münkler, Durch den Sehschlitz. Man mache sich nichts vor: Der Westen kann den Krieg in Afghanistan nicht gewinnen, in: Süddeutsche Zeitung, 6.4.2009, S. 11.

25 Christiane Fröhlich et al. (Hg.), Friedensgutachten 2010, Münster 2010, Vorwort der Herausgeberinnen und Herausgeber.

26 Gerhard Beestermöller, Kirche und Krieg. Wann dürfen Soldaten kämpfen? Was die christliche Ethik zum Einsatz der Bundeswehr in Afghanistan sagt, in: Der Tagesspiegel, 29.1.2010.

27 Zitiert in Aryn Baker, Afghan Women and the Return of the Taliban, Time Magazine, 29.7.2010 (Online-Version). http://www.time.com/time/world/ article/0,8599,2007238,00.html (24.8.2010).

28 Richard Herzinger, „Die Wirtschaftskrise ist eine Menschenrechtskrise“, 27.5.2010, http://www.welt.de/die-welt/politik/article7801142/DieWirtschaftskrise-ist-eine-Menschenrechtskrise.html (28.5.2010).

29 Ein Scheitern in Afghanistan, so diese Argumentationslinie, hätte also allenfalls geringe Aussagekraft darüber, wie es um die Zukunft des liberalen Peacebuilding bestellt ist. In der Tat ergeben sich aus der spezifischen Lage in Afghanistan Anforderungen an ISAF, die höher sind als in anderen Einsatzgebieten, in denen ambitionierte friedensschaffende Missionen unternommen wurden. Vgl. z.B. David Kilcullen, The Accidental Guerilla. Fighting Small Wars in the Midst of a Big One, New York 2009, S. 39-114, bes. S. $109-114$. 
dukusch verteidigt wird. “30 Deshalb wird auch US-Präsident Barack Obama nicht müde, Afghanistan als „war of necessity“ zu beschreiben (und ihn damit vom „war of choice“ im Irak, den er ablehnte, abzugrenzen): „So this is not only a war worth fighting. This is fundamental to the defense of our people."31 Andere Regierungschefs haben den Einsatz in ähnlicher Weise mit der Verteidigung eigener vitaler Sicherheitsinteressen erklärt und gerechtfertigt. Im Gegensatz zu vielen UN-geführten Friedensmissionen der letzten beiden Jahrzehnte, bei denen die internationale Gemeinschaft vor allem aus solidarischen und humanitären Motiven handelte, dominierten bei der Entscheidung zur Intervention in Afghanistan also unter dem Eindruck der Anschläge vom 11. September 2001 neu formulierte Sicherheitsinteressen. Dennoch gelingt es den politischen Eliten in liberalen Demokratien kaum, politische Unterstützung für diese angeblich so wichtige Mission aufrechtzuerhalten.

Demokratische Gesellschaften stehen zwei grundlegenden Dilemmata gegenüber. Das erste Dilemma bezeichnet Gil Merom als „balance-of-tolerance“. ${ }^{32} \mathrm{Um}$ in einem Umfeld wie in Afghanistan die Aufständischen soweit zurückzudrängen, dass sie den Staatsaufbau nicht mehr entscheidend torpedieren können, müssen die truppenstellenden Staaten bereit sein, möglicherweise nur schwer akzeptable Strategien und Entwicklungen mitzutragen: „[S]tates need their soldiers to be ready to harm others and to be killed or maimed, and their citizens to accept the army's behavior and the risks their kin in arms face. Achieving a certain balance between these two requirements - the readiness to bear the cost of war and the readiness to exact a painful toll from others - is a precondition for succeeding in war."33 Wie Merom zeigt, fällt dies liberalen Demokratien besonders schwer. Das zweite Dilemma könnte als liberales Dilemma bezeichnet werden. Ein Afghanistan, das nicht einmal liberal-demokratischen/menschenrechtlichen Mindeststandards gerecht wird, lässt sich einem „liberalen Gewissen “34 kaum vermitteln - wie lässt sich der Tod eigener Soldaten rechtfertigen, wenn sie nur beim Aufbau eines illiberalen Regimes mitgewirkt haben, das möglicherweise nicht einmal fundamentale Menschenrechte achtet ${ }^{35}$ Gleichzeitig aber stehen truppenstellende Gesellschaften dem Einsatz massiver Mittel, die wohl notwendig wären, um die Voraussetzung für den Erfolg liberal-demokratischer Strukturen zu schaffen, sehr skeptisch gegenüber.

30 Merkel, Angela, Regierungserklärung zum Einsatz der Bundeswehr in Afghanistan, 22.4.2010, http://www.bundesregierung.de/nn_1502/Content/ DE/Regierungserklaerung/2010/2010-04-22-merkel-erklaerung-afghanistan. html (19.5.2010).

31 Barack Obama, Remarks at the Veterans of Foreign Wars Convention, 17.8.2009, http://www.whitehouse.gov/the_press_office/remarks-by-thepresident-at-the-veterans-of-foreign-wars-convention (22.5.2010)

32 Gil Merom, How Democracies Lose Small Wars: State, Society, and the Failures of France in Algeria, Israel in Lebanon, and the United States in Vietnam, Cambridge 2003, S. 19.

33 Ebd., S. 19. Für eine Weiterentwicklung des Arguments siehe Lorenzo Zambernardi, Counterinsurgency's Impossible Trilemma, in: The Washington Quarterly, Vol. 33, No. 3, 2010, S. 21-34.

34 Vgl. Michael Howard, War and the Liberal Conscience, London 2008 [1977].

35 So z. B. Valerie Hudson und Patricia Leidl: "American women are right to ask why we are sending their sons and daughters to fight and die so that Afghan women can continue to be treated like an inferior subspecies of humanity. When you break faith with Afghan women, you break faith with American women. You also break faith with your reasons for being there in the first place." Valerie Hudson und Patricia Leidl, Betrayed. They said we were there to save Afghanistan's women. So how come we haven't?, 10.5.2010, http://www. foreignpolicy.com/articles/2010/05/07/the_us_is_abandoning_afghanistan s_women (3.9.2010).
Obwohl die Gefahr für die internationale Sicherheit, die von zerfallenden Staaten ausgehen kann, nach den Terroranschlägen des 11. Septembers 2001 stärker ins öffentliche Bewusstsein rückte und die Stabilität von „Afpak“ als zentrales Sicherheitsinteresse definiert wird, ist die Unterstützung für die ISAF-Mission in den westlichen Ländern, die Truppen stellen, schon heute bemerkenswert niedrig. In Deutschland sprachen sich im Mai 201065 Prozent der Befragten einer Allensbach-Umfrage dagegen aus, „dass sich deutsche Soldaten auch in Zukunft an der Schutztruppe in Afghanistan beteiligen“ . ${ }^{36}$ In den Niederlanden hat der Streit über eine über 2010 hinausgehende Beteiligung an ISAF die Koalition von Ministerpräsident Balkenende zu Fall gebracht. Der neue britische Verteidigungsminister Liam Fox nannte Afghanistan „ein zerbrochenes Land aus dem 13. Jahrhundert“, man müsse das Engagement dort jeden Tag aufs Neue hinterfragen. ${ }^{37}$ Und in den USA hat Präsident Obama seiner Bevölkerung versprochen, im Juli 2011 mit dem Rückzug amerikanischer Truppen zu beginnen. ${ }^{38} \mathrm{Ob}$ die Lage in Afghanistan zu diesem Zeitpunkt tatsächlich einen solchen Rückzug und eine schrittweise erfolgende Übergabe der Provinzen an ausschließlich afghanische Sicherheitskräfte zulässt, ohne dass radikale Kräfte das entstehende Machtvakuum füllen, wird also möglicherweise kein entscheidendes Kriterium mehr sein. In derselben Rede beteuerte Obama zwar Amerikas fortgesetztes Engagement für die Menschenrechte. Doch vieles spricht dafür, dass ein voreiliger Rückzug und Zugeständnisse in Verhandlungen mit radikalen Kräften fatale Konsequenzen für die menschenrechtliche Situation der afghanischen Bevölkerung hätten. Zwei Beispiele aus dem August 2010 illustrieren diese möglichen Folgen: Im Gebiet des Regionalkommandos Nord wurden erstmals seit dem Ende der Talibanherrschaft 2001 zwei Menschen wegen einer außerehelichen Affäre gesteinigt. ${ }^{39} \mathrm{Zu}$ dem sorgte das Cover einer Ausgabe des Time Magazine für Aufsehen, auf dem das Portrait einer 18-jährigen afghanischen Frau abgebildet wurde, der nach dem Urteil eines Taliban-Richters die Nase und die Ohren abgetrennt worden waren. Der Titel der Ausgabe lautete, bewusst ohne Fragezeichen: „What happens if we leave Afghanistan“. ${ }^{40}$

\section{Mögliche Folgen für liberale Ordnungspolitik: „Internationale Gemeinschaft" in einer „Welt ohne den Westen"?}

Es mag bisweilen problematisch sein, von einem spezifischen Einzelfall auf generelle Entwicklungen zu schließen. Trotz aller Besonderheiten Afghanistans legt die Entwicklung dort jedoch

36 Thomas Petersen, Wird Deutschland am Hindukusch verteidigt?, in Frankfurter Allgemeine Zeitung, 26.5.2010, S. 5.

37 Rachel Sylvester und Alice Thomson, True Blue Tory Flying the Flag for Our Boys and Eurosceptics, The Times, 21.5.2010, S. 62-63, S. 62. Übersetzung der Autoren.

38 Barack Obama, Remarks in Address to the Nation on the Way Forward in Afghanistan and Pakistan, 1.12.2009, http://www.whitehouse.gov/thepress-office/remarks-president-address-nation-way-forward-afghanistan-andpakistan (25.5.2010).

39 Agnes Tandler, Rückkehr der Taliban befürchtet, in: die tageszeitung (taz), 23.8.2010, http://www.taz.de/1/politik/asien/artikel/1/rueckkehr-der-talibanbefuerchtet/ (25.8.2010). Eine Woche vorher war Berichten zufolge in der nordwestlichen Provinz Badghis eine schwangere Witwe wegen Ehebruchs ausgepeitscht und erschossen worden.

40 Time Magazine, 9.8.2010. 
nahe, dass sowohl der Glaube an die „Exportfähigkeit“ demokratischer Werte als auch die Bereitschaft westlicher Länder, für diese Aufgabe auch militärische Risiken einzugehen, mit einem möglichen „afghanischen Trauma“ oder einem „Afghanistan-Syndrom “41 einen herben Dämpfer erleiden könnten. Sollten sich in Afghanistan nach Abzug der ISAF-Schutztruppe erneut politische Strukturen etablieren, die liberal-demokratischen Standards entgegenstehen, würde nicht nur die Idee, dass diese Normen universell sind, sondern auch der Gedanke, dass externe Akteure ihre Durchsetzung vorantreiben können und sollen, beträchtlichen Schaden nehmen. Vergleiche mit Somalia 1993/94 sind zwar aus vielen Gründen problematisch und unzureichend. Doch wenn schon das Scheitern der Friedensmission UNOSOM II nach dem amerikanischen Rückzug die Wirkmächtigkeit liberaler Normen schwächte ${ }^{42}$ - mit katastrophalen Folgen etwa in Ruanda ${ }^{43}$ - , kann man ermessen, welche Konsequenzen ein Scheitern in Afghanistan, wo die internationale Gemeinschaft ein Vielfaches investiert hat, für liberale Ordnungsvorstellungen haben könnte. Es wäre ein vorläufiger Kulminationspunkt mehrerer Entwicklungen, die zu einer Schwächung des liberalen Moments beigetragen haben.

Zum einen sind die Gründe für diese Schwäche in den Ambivalenzen des Liberalismus selbst zu finden, die sich in verschiedenen Ausprägungen zwischen den Idealtypen eines „Liberalism of Restraint“ und eines „Liberalism of Imposition“ ausdrückt. ${ }^{44}$ Ein zu sehr gezügelter Liberalismus wurde vor allem in der Tatenlosigkeit gegenüber mehreren Völkermorden in den letzten beiden Jahrzehnten manifest, welche die noch junge Norm der humanitären Intervention in Frage stellte. ${ }^{45}$ Die fatalen Auswüchse des „Liberalism of Imposition“ dagegen waren im Irakkrieg $2003^{46}$ und der „Freedom Agenda“47 der Regierung von George W. Bush besonders offensichtlich, welche die Begriffe von „Freiheit“ und „Demokratie“ und damit die dahinterstehenden Prinzipien ad absurdum geführt und nachhaltig diskreditiert haben. Bruce Russett mag sich zwar auf einen unnötigen Vergleich eingelassen haben, als er schrieb, dass sich viele Vertreter des Demokratischen Friedens nach Irak wohl wie die Atomphysiker 1945 fühlten, weil ihre „Kreationen“ derart pervertiert worden seien. ${ }^{48}$ Aber er macht doch deutlich, wie einschneidend die Zäsur des Irakkriegs für die Verbreitung und die Legitimität liberaler Ideen war, freilich

41 Vgl. Eliot A. Cohen, Will There Be an Afghanistan Syndrome?, in: The Washington Post, 27.6.2010, S. B1.

42 Nicholas Wheeler, Saving Strangers. Humanitarian Intervention in International Society, New York 2000, S. 215f. und 224f.

43 Samantha Power, „A Problem from Hell”. America and the Age of Genocide, New York 2007, S. 377-380.

44 Georg Sørensen, Liberalism of Restraint and Liberalism of Imposition: Liberal Values and World Order in the New Millennium, in: International Relations, Vol. 20, No. 3, 2006, S. 251-272

45 Vgl. z. B. Thomas Weiss, The Sunset of Humanitarian Intervention? The Responsibility to Protect in a Unipolar Era, in: Security Dialogue, Vol. 35, No. 2, 2004, S. 135-153.

46 Für eine liberale Verteidigung des Irakkriegs siehe z. B. Fernando Tesón, Ending Tyranny in Iraq, in: Ethics \& International Affairs, Vol. 19, No. 2, 2005 , S. 1-20.

47 Vgl. v.a. George W. Bush, Second Inaugural Address, Washington, DC, 20.1.2005; siehe auch Natan Sharansky mit Ron Dermer, The Case for Democracy: The Power of Freedom to Overcome Tyranny and Terror, New York 2004, ein Buch, das Bush offenbar nachhaltig beeindruckte.

48 Bruce Russett, Bushwhacking the Democratic Peace, in: International Studies Perspectives, Vol. 6, No. 4, 2005, S. 395-408, S. 396: "Many advocates of the democratic peace may now feel rather like many atomic scientists did in 1945." Vgl. weiterführend die Beiträge in Anna Geis, Harald Müller und Wolfgang Wagner (Hg.), Schattenseiten des Demokratischen Friedens. Zur Kritik einer Theorie liberaler Außen- und Sicherheitspolitik, Frankfurt am Main 2007. nicht nur in der Wahrnehmung von Politikwissenschaftlern, sondern auch von vielen Millionen Menschen weltweit, die einer amerikanischen Hegemonie möglicherweise ohnehin skeptisch gegenüberstehen. Obwohl sich die US-Intervention im Irak in vielerlei Hinsicht deutlich von den Friedensmissionen mit UN-Mandat unterscheidet, ${ }^{49}$ hat das Desaster des Irakkriegs möglicherweise gar „die Plausibilität und Rechtfertigung internationaler Interventionen schlechthin untergraben, wie wünschenswert und gut zu rechtfertigen sie in anderen Fällen auch sein mögen. “50

Zum anderen gerät der liberale Moment zunehmend von außen unter Druck. Einige Kommentatoren haben bereits eine Rückkehr des autoritären Staates und damit eine „Rückkehr der Geschichte "51 diagnostiziert, die liberale Ordnungsvorstellungen auf fundamentale Weise herausfordern wird. Ob man tatsächlich von einem Wiederaufstieg autoritärer Mächte sprechen kann, ${ }^{52}$ welche die Werte der liberalen Demokratie nicht nur ablehnen, sondern zunehmend ein anderes Ordnungsmodell zu propagieren suchen, wird eine Aufgabe künftiger Forschung sein. ${ }^{53}$ Bereits jetzt sprechen einige Beobachter von der Entstehung einer „Welt ohne den Westen“, deren grundlegende Ordnungsmuster einem eher traditionellen Souveränitätsverständnis folgen. ${ }^{54}$ Wolfgang Seibel geht davon aus, dass sich das „Fenster der Gelegenheit“ für die Einrichtung umfassender Friedensmissionen bis hin zu Übergangsverwaltungen unter dem Dach der Vereinten Nationen bereits wieder geschlossen hat. Der (Wieder-)Aufstieg insbesondere von Russland und China habe dazu geführt, dass die westlichen Länder kaum mehr in der Lage seien, die institutionellen Mechanismen der Vereinten Nationen für die Verbreitung westlicher Standards einzusetzen. ${ }^{55}$ Insgesamt scheint als Ergebnis dieser Entwicklungen die Ausrichtung weltpolitischen Handelns an liberalen Ordnungsvorstellungen keineswegs mehr so selbstverständlich, wie dies in den Jahren nach dem Ende des Ost-West-Konflikts der Fall gewesen ist.

An dieser Stelle könnte man die Frage aufwerfen, ob selbst ein „Erfolg“ in Afghanistan noch in der Lage sei, den liberalen Moment zu retten - oder ob dessen Ende nicht ohnehin unaufhaltsam ist. Aus dieser Perspektive wäre ein „Scheitern“ in Afghanistan nur noch der finale Sargnagel. ${ }^{56}$ Sofern diese Hypothese impliziert, dass damit die normativen Entwicklungen der letzten Jahrzehnte zur Disposition stehen, verkennt sie jedoch die weitreichende Transformation der Weltpolitik im Zuge des

49 Dazu ausführlich Paris, Saving Liberal Peacebuilding, a.a.O., S. 345-346.

50 Wolfgang Seibel, ModerneProtektorate als Ersatzstaat: UN-Friedensoperationen und Dilemmata internationaler Übergangsverwaltungen, in Gunnar F. Schuppert und Michael Zürn (Hg.), Governance in einer sich wandelnden Welt, Wiesbaden 2008, S. 499-530, S. 514

51 Robert Kagan, The Return of History and the End of Dreams, London 2008.

52 Vgl. z.B. Azar Gat, The Return of Authoritarian Great Powers, in: Foreign Affairs, Vol. 86, No. 4, 2007, S. 59-69.

53 Vgl. bereits Julia Bader und Antje Kästner, Mehr Autokratie wagen? Russland und China als Konkurrenten westlicher Demokratieförderer, in: Internationale Politik, Vol. 65, No. 3, 2010, S. 32-36; Peter Burnell, Is There a New Autocracy Promotion?, FRIDE Working Paper No. 96, Madrid 2010.

54 Siehe für eine erste Formulierung der These Naazneen Barma, Ely Ratner und Steven Weber, A World Without the West, in: The National Interest, No. 90 2007, S. 23-30; für erste empirische Belege Naazneen Barma, Giacomo Chiozza, Ely Ratner und Steven Weber 2009: A World Without the West? Empirical Patterns and Theoretical Implications, in: Chinese Journal of International Politics, Vol. 2, No. 4, 2009, S. 525-544.

55 Seibel, Moderne Protektorate als Ersatzstaat, a.a.O., S. 512-513.

56 Diese Formulierung verdanken die Autoren einer anonymen Gutachterin oder einem anonymen Gutachter. 
liberalen Moments. Vieles deutet eher darauf hin, dass sich die Spannung zwischen einem „Liberalism of Restraint“ und einem „Liberalism of Imposition“ - deren Bewältigung nach Georg Sørensen eine entscheidende Bedingung für wirklichen Fortschritt in Richtung einer liberalen Weltordnung ist ${ }^{57}$ - wieder zugunsten eines „Liberalism of Restraint" verschiebt. Ein Scheitern in Afghanistan wäre das bisher deutlichste Zeichen dieser Verschiebung - und damit von hohem symbolischen Charakter für die zukünftige Gestaltungskraft westlicher Ordnungspolitik.

Zu den Zweifeln gegenüber der Sinnhaftigkeit internationaler Interventionen dürften zudem massive Ressourcenprobleme treten, die durch die globale Finanz- und Wirtschaftskrise noch verschärft wurden. Haushaltsausgaben für Außen-, Entwicklungs- und Sicherheitspolitik werden auf Jahre hinaus nachhaltig unter Druck geraten. Dies wird auch für das Leitbild einer „internationalen Gemeinschaft“, die für sich in Anspruch nimmt, einem Verständnis von human security zu folgen, weitreichende Folgen haben. Schließlich sind die Vereinten Nationen auf die militärischen Beiträge der westlichen Demokratien angewiesen, um durchsetzungsfähige Friedensmissionen durchführen zu können. Aus diesem Grund hat der frühere Generalsekretär Kofi Annan auch das zunehmend globale Engagement der NATO begrüßt: „Looking to the future, NATO's increasing willingness to ,go global' presents important opportunities, in particular for Africa. [...] It is also likely that the year ahead will see other new peace operations in Africa, as well as in Haiti and possibly elsewhere. Should such a surge take place, stronger support from NATO would be tremendously helpful. Specifically, NATO might be employed in a ,peace enforcement' role [...] before the deployment of a UN operation. "58 Es ist jedoch davon auszugehen, dass ein Scheitern in Afghanistan einer Weiterentwicklung der globalen Rolle der NATO einen signifikanten Dämpfer versetzen würde. ${ }^{59}$ Berücksichtigt man zudem, dass die Unterstützung westlicher Länder für UN-geführte Friedensmissionen ohnehin zurückgegangen ist, obwohl die Missionen „westlicher“ geworden sind als je zuvor, ${ }^{60}$ bleiben eher trübe Aussichten für zukünftige Missionen in Ländern, in denen externe Unterstützung dazu beitragen könnte, menschliches Leid zu lindern. Nicht zuletzt werden darunter gerade diejenigen Bevölkerungen zu leiden haben, deren Lage von geringerer strategischer Bedeutung für die „internationale Gemeinschaft“ ist. Die Selektivität humanitären Eingreifens wird somit eher steigen, was für die Geltungskraft liberaler Normen mit universellem Anspruch eine massive Herausforderung darstellen wird. Ähnliches gilt auch für die De-

57 Sørensen, Liberalism of Restraint and Liberalism of Imposition, a.a.O., S. 264: "[I]t would appear that the Liberalism of Restraint is too little; it does not promise to deal effectively with vast problems of human anguish and distress; it is potentially a recipe for inaction. The Liberalism of Imposition would appear to be too much; it seriously threatens to undermine the very liberal values that it seeks to promote; it is potentially a recipe for overreaction. That is the core tension in liberalism: finding a way of mastering it is an inescapable condition for further real progress towards a liberal world order."

58 Kofi Annan, Secretary-General's Opening Remarks at Meeting With Nato Parliamentarians, 8.3.2004, http://www.un.org/apps/sg/sgstats.asp?nid=808 (24.5.2010)

59 Timo Noetzel und Benjamin Schreer, NATO's Vietnam? Afghanistan and the Future of the Atlantic Alliance, in: Contemporary Security Policy, Vol. 30, No. 3, 2009, S. 529-547, S. 543f.

60 Vgl. zu diesem Paradox v.a. Alex J. Bellamy und Paul D. Williams, The West and Contemporary Peace Operations, in: Journal of Peace Research, Vol. 46, No. 1, 2009, S. 39-57. mokratieförderung oder Menschenrechtspolitik, die im Zuge einer sich verstärkenden Ressourcenproblematik ${ }^{61}$ aller Voraussicht nach von gegenläufigen Interessen an der verstärkten Kooperation mit autokratischen Regimen in den Hintergrund gedrängt werden könnten. In der Summe mögen diese Entwicklungen dazu führen, dass die weitreichenden Entwicklungen in Richtung des solidaristischen Flügels der English School von einer Gegenbewegung konterkariert werden, die mit der Relativierung politischer Ordnungsvorstellungen in unterschiedlichen Kontexten eher der Interpretation des pluralistischen Lagers entspricht.

\section{Schlussbetrachtung: Das Ende des liberalen Moments?}

Eine sehr populäre Deutung der derzeitigen Lage bedient sich der Vorstellung von Afghanistan als einem „graveyard of empires“62. Diese Deutung impliziert zum einen, dass auch die amerikanische Supermacht unweigerlich an der unmöglichen Aufgabe in Afghanistan scheitern werde. Ein solches „Scheitern" der USA und ihrer Bündnispartner - wie auch immer sich die Definitionen von „Erfolg“von heute bis zum Zeitpunkt des Rückzugs noch ändern werden - liegt mittlerweile im Bereich des Möglichen. Zum anderen suggeriert die Deutung vom Friedhof der Imperien aber auch, dass ein Scheitern in Afghanistan einen schweren Rückschlag für das amerikanische „Empire“ mit sich bringen würde. Noch viel mehr als der Status der USA als Supermacht ist in Afghanistan aber die Dominanz liberaler Ordnungsvorstellungen gefährdet. Man kann dieser Entwicklung etwas Positives abgewinnen, wenn damit die Abkehr von der liberalen Hybris einhergeht, die zumindest in der Vorbereitung des Irakkriegs unübersehbar war. Dennoch wäre ein nachlassendes Eintreten der westlichen Demokratien für liberale Werte der Weiterentwicklung der „internationalen Gemeinschaft" in Richtung einer Solidargemeinschaft alles andere als zuträglich. Denn während ein Großteil der Kritik an der Praxis des liberal peacebuilding berechtigt ist, ist es angesichts bestehender Erfolge und aufgrund des Mangels an Alternativen weder angebracht noch moralisch tragfähig, die Sinnhaftigkeit solcher Missionen gänzlich in Frage zu stellen. Schließlich käme dies der Entscheidung gleich, Millionen von Menschen ihrem Schicksal zu überlassen. ${ }^{63}$

Obwohl die Ressourcen der westlichen Demokratien - insbesondere im Kontext der globalen Wirtschaftskrise - ohnehin begrenzt sind und eine gewisse Selektivität in den internationalen Interventionen, die oftmals als Inkonsistenz oder gar Scheinheiligkeit in der Menschenrechtspolitik ausgelegt wird, generell nicht zu vermeiden ist, ${ }^{64}$ hat der Einsatz in Afghanistan wohl gerade deswegen eine weitreichende symbolische Wir-

61 Siehe hierzu die Beiträge zum Themenschwerpunkt „Sicherheitsaspekte zukünftiger Rohstoffversorgung" in Sicherheit + Frieden, Vol. 27, No. 4.

$62 \mathrm{Vgl}$. z.B. Seth Jones, In the Graveyard of Empires. America's War in Afghanistan, Oxford/New York 2009; David Isby, Afghanistan: Graveyard of Empires. A New History of the Borderlands, New York 2010.

63 Vgl. insbesondere Paris, Saving Liberal Peacebuilding, a.a.O., v.a. S. 338 u. 360.

64 Für eine nuancierte Diskussion der Selektivitätsproblematik vgl. v.a. Martin Binder, Humanitarian Crises and the International Politics of Selectivity, in: Human Rights Review, Vol. 10, No. 3, 2009, S. 327-348. 
kung. Schließlich handelt es sich hier um einen Fall, der von den politischen Eliten in den westlichen Ländern als zentral für die eigene Sicherheit verstanden wird und für dessen Lösung die internationale Gemeinschaft bereits massive Ressourcen aufgewendet hat. Wenn nun selbst dieses Land in einem $\mathrm{Zu}$ stand zurückgelassen würde, der höchstwahrscheinlich eine katastrophale Menschenrechtssituation nach sich zöge, würde nicht zuletzt die Aussicht auf ein Eingreifen externer Akteure in jenen Situationen sinken, in denen diese über keine eng verstandenen Sicherheitsinteressen verfügen. Zudem würde ein solcher Rückzug die ganz essentielle Frage aufwerfen, wie viel der „internationalen Gemeinschaft“ und vor allem den liberalen westlichen Demokratien die vielgepriesenen liberalen Werte tatsächlich wert sind. Sollte in Afghanistan also etwas zu Grabe getragen werden, dann könnte dies die westliche Überzeugung sein, dass der „liberale Moment“ aktiv durch Weltordnungspolitik getragen und unterstützt werden sollte. Er wäre dann tatsächlich nur ein „Moment“ gewesen.

\title{
Afghanistan - Ein langer Weg zur Rechtsstaatlichkeit
}

\author{
Julia Pfeiffer*
}

\begin{abstract}
Since 2001 the international community supports the Afghan state in building the rule of law in the country. Although some progress has been made, the rule of law is still weak. However, as the paper shows, the situation of decreasing security and lack of the rule of law are interrelated and affect each other in a negative way. Therefore, strengthening the rule of law should be the priority in the ongoing state-building process. As long as the Afghan government cannot provide a stable and secure environment based on the principles of the rule of law, reconciliation and peace cannot be achieved. Afghan authorities must finally start to accept and implement the concepts of rule of law by ceasing injustice, impunity and corruption throughout the country. The new "Prioritization and Implementation Plan", which the Afghan government presented on the International Kabul Conference in July 2010, may be a first step in this direction.
\end{abstract}

Keywords: Afghanistan, state-building, rule of law and stability, judicial reform

Afghanistan, State-Building, Rechtsstaatlichkeit und Stabilität, Justizreform

\section{Einleitung}

$\mathrm{Z}$ um Wiederaufbau Afghanistans, der seit nunmehr fast neun Jahren andauert, gehört auch die Schaffung eines stabilen Rechtsstaats. Dieses Ziel ist auch in der Präambel der neuen Verfassung Afghanistans von 2004 vorgesehen: „Wir das Volk von Afghanistan, haben (...) zur Errichtung einer Zivilgesellschaft (...) basierend auf Rechtsstaatlichkeit, sozialer Gerechtigkeit, dem Schutz der Menschenrechte und der Menschenwürde (...) diese Verfassung (...) verabschiedet.“ Auch wenn das Rechtsstaatsprinzip im Verfassungstext selbst nicht ausdrücklich genannt wird, so kann es aus verschiedenen Vorschriften abgeleitet werden.

Der folgende Artikel befasst sich mit der Frage, in welchem Umfang Rechtsstaatlichkeit in Afghanistan vorhanden ist und führt weiter aus, inwieweit ein Zusammenhang zwischen Rechtsstaatlichkeit, Stabilität und Sicherheit hergestellt werden kann. Beleuchtet werden außerdem die letzten Entwicklungen beim Staatsaufbau in Afghanistan seit dem Sommer 2010.

\section{Rechtsstaatlichkeit in Afghanistan?}

Rechtsstaatlichkeit setzt sich aus fünf Prinzipien zusammen: Rechtsbindung der Gewalten, Gleichheit vor dem Gesetz, Ge- währleistung der öffentlichen Ordnung, eine effiziente und unabhängige Justiz sowie Achtung der Menschenrechte. ${ }^{1}$

Obwohl die Gesetzesbindung der Exekutive in der afghanischen Verfassung (AV) in den Artikeln 50, 63, 76 und 77 vorgesehen ist, setzt sich die Exekutive immer wieder über dieses Prinzip hinweg. So geschehen zum Beispiel beim Misstrauensvotum gegen den ehemaligen Außenminister Spanta aus dem Jahr 2007, das von Präsident Karzai ignoriert wurde. ${ }^{2}$ Spanta blieb bis Januar 2010 Außenminister.

Die Gerichte sollen laut Artikel 130 der afghanischen Verfassung ihre Urteile auf Grundlage der Verfassung und Gesetze fällen. Dies geschieht in vielen Fällen jedoch nicht. Der Grund hierfür ist insbesondere die weit verbreitete Korruption in der Richter- und Staatsanwaltschaft. ${ }^{3}$ Parteilichkeit und Bestech-

* Die Autorin ist Mitarbeiterin der Afghanistan-Projekte des Max-Planck-Instituts für ausländisches öffentliches Recht und Völkerrecht (jpfeiffe@mpil.de). Die im Text geäußerte Meinung ist allein die der Autorin.

1 Rachel Kleinfeld "Competing Definitions of the Rule of Law" in Thomas Carothers, "Promoting the Rule of Law Abroad - In Search of Knowledge", Carnegie Endowment for International Peace, Washington D.C., 2006, S. 31 ff. Im Folgenden stützt sich die Autorin auf diese Ansätze. Zur akademischen Diskussion in diesem Bereich siehe Kleinfeld S. 32, Fn 10

2 Siehe Ramin Moschtaghi "Aktuelle Probleme beim Rechtsstaatsaufbau in Afghanistan: Das Gutachten des Obersten Gerichtshofs zum Misstrauensantrag des Unterhauses gegen den Außenminister" in ZaöRV 68 (2002) 2, S. 509 ff.

3 UNDOC Report: Corruption in Afghanistan - Bribery as reported by the Victims, January 2010, S. 27f. (http://www.reliefweb.int/rw/RWFiles2010. nsf/FilesByRWDocUnidFilename/FBUO-7ZUKNH-full_report.pdf/\$File/ full_report.pdf (10. September 2010). 